\title{
The BeppoSAX 0.1-18 keV spectrum of the bright atoll source GX 9+1: an indication of the source distance
}

\author{
R. Iaria ${ }^{1}$, T. Di Salvo ${ }^{1}$, N. R. Robba ${ }^{1}$, G. Lavagetto ${ }^{1}$, L. Burderi ${ }^{1,2}$, L. Stella ${ }^{2}$, and M. van der Klis ${ }^{3}$ \\ 1 Dipartimento di Scienze Fisiche ed Astronomiche, Università di Palermo, via Archirafi 36, 90123 Palermo, Italy \\ e-mail: iaria@fisica.unipa.it \\ 2 Osservatorio Astronomico di Roma, via Frascati 33, 00040 Monteporzio Catone (Roma), Italy \\ 3 Astronomical Institute "Anton Pannekoek", University of Amsterdam and Center for High-Energy Astrophysics, \\ Kruislaan 403, NL 1098 SJ Amsterdam, The Netherlands
}

\author{
Received 22 October 2004 / Accepted 5 March 2005
}

\begin{abstract}
We report the results of a long, $350 \mathrm{ks}$, BeppoSAX observation of the bright atoll source GX 9+1 in the $0.12-18 \mathrm{keV}$ energy range. During this observation GX 9+1 showed a large count rate variability in its lightcurve. From its color-color diagram we selected six zones and extracted the source energy spectrum from each zone. We find that the model, composed of a blackbody plus a Comptonized component absorbed by an equivalent hydrogen column of $\sim 1.4 \times 10^{22} \mathrm{~cm}^{-2}$, fits the spectra in the energy range $1-18 \mathrm{keV}$ well; however, below $1 \mathrm{keV}$ a soft excess is present. We find that the spectrum of GX 9+1, in the $0.12-18 \mathrm{keV}$ energy range, is well fitted by the model above, if we use an equivalent hydrogen column of $\sim 0.8 \times 10^{22} \mathrm{~cm}^{-2}$, together with several absorption edges from ionized matter (O VII, O VIII, Ne IX, Ar XVII, and Ca XX) and an absorption line from Si XIV. From the study of these features we deduce that the electron density of the plasma and the equivalent hydrogen column density associated with the ionized matter is $\sim 10^{12} \mathrm{~cm}^{-3}$ and $\sim 10^{23} \mathrm{~cm}^{-2}$, respectively, at a distance from the central object of $r \sim 10^{11} \mathrm{~cm}$, while at larger distances the equivalent hydrogen column density associated to the ionized matter decreases down to $\sim 10^{22} \mathrm{~cm}^{-2}$.

The value of the equivalent hydrogen column of $\sim 0.8 \times 10^{22} \mathrm{~cm}^{-2}$ associated to the interstellar matter implies that GX $9+1$ is at a distance of $5 \mathrm{kpc}$, and not $8.5-10 \mathrm{kpc}$ as usually assumed. This result implies that the atoll source GX 9+1 does not emit at the Eddington limit, as supposed until now, but has a luminosity of $6 \times 10^{37} \mathrm{erg} / \mathrm{s}$ in the $0.1-18 \mathrm{keV}$ energy range, typical of LMXBs belonging to the bright atoll class.
\end{abstract}

Key words. accretion, accretion discs - stars: individual: GX 9+1 - stars: neutron - X-ray: stars - X-ray: binaries X-ray: general

\section{Introduction}

The first observation of GX 9+1 (4U 1758-205) was carried out in 1966 using a proportional counter sensitive to $1.5-10 \mathrm{keV}$ $\mathrm{X}$-rays on board a rocket (Gursky et al. 1967). Two years later the presence of the source was confirmed by Bradt et al. (1968), who also measured the source position at a Galactic longitude of $9.1^{\circ}$ and a Galactic latitude of $1.2^{\circ}$, well in the Galactic bulge. The distance and companion star of GX 9+1 have remained unknown, despite several attempts made at identifying the optical counterpart in the early ' 70 s.

Using the Medium Energy (ME) instrument on board EXOSAT, Langmeier et al. (1985) performed a spectral and timing analysis of an observation of GX 9+1 in the 1-30 keV energy band. During the observation, the source flux varied irregularly on time scales from minutes to hours. No periodic signal in the range from $16 \mathrm{~ms}$ to $2000 \mathrm{~s}$ was found with an upper limit of around $1 \%$ ( $3 \sigma$ confidence level) on the pulsed fraction. The hardness ratio ([3.4-7.5 $\left.\mathrm{keV}] /\left[\begin{array}{lll}1-3.2 \mathrm{keV}\end{array}\right]\right)$ showed intensity correlated changes. The energy spectrum was fitted by a double component model, a blackbody component $(k T=1.16-1.26 \mathrm{keV})$ that was perhaps produced in a region near the neutron star surface, together with a thermal bremsstrahlung $(k T=13-15 \mathrm{keV})$. No iron line at $6.4-6.7 \mathrm{keV}$ was detected. The X-ray spectral behavior of GX 9+1 indicated that this source belongs to the class of Low-Mass Xray Binaries (LMXBs). White et al. (1988) reanalysed the EXOSAT ME energy spectrum fitting the data with a blackbody component $(k T=1.5 \mathrm{keV})$, plus a Comptonized component with an electron temperature of $3 \mathrm{keV}$. Both these components were absorbed by neutral matter having an equivalent hydrogen column of $2.1 \times 10^{22} \mathrm{~cm}^{-2}$. The extrapolated luminosities in the $0.1-30 \mathrm{keV}$ energy band associated to the blackbody and the Comptonized component were $6 \times 10^{37} \mathrm{erg} / \mathrm{s}$ and $1.7 \times 10^{38} \mathrm{erg} / \mathrm{s}$, respectively, assuming a distance to the source of $10 \mathrm{kpc}$. The distance of $10 \mathrm{kpc}$ was an upper limit, because 
the source is in the direction of the Galactic center. Despite all this, GX 9+1 has still been assumed since to be a high luminous $\mathrm{X}$-ray source.

Hasinger \& van der Klis (1989), when studying the fast timing properties of GX 9+1 based on the EXOSAT data, concluded that the source was to be included in the Atoll class. GX $9+1$ forms the subclass of persistently bright atoll sources together with GX 3+1, GX 9+9, and GX 13+1. In their Color-Color Diagram (CD), these sources have been seen to trace out only the banana branch, and their power spectra (PSD) can be described by relatively strong $(\sim 3.5 \% \mathrm{rms})$ Very Low Frequency Noise (VLFN) and weak ( $2.5 \% \mathrm{rms})$ High Frequency Noise (HFN), as compared to other Atoll sources (Schulz et al. 1989). An RXTE observation of the source (Wijnands et al. 1997) showed that, similar to the other bright Atolls, GX 9+1 did not show Quasi Periodic Oscillations (QPOs) at $\mathrm{kHz}$ frequencies in its power spectrum. This was in agreement with the fact that Atoll sources in general do not show kHz QPOs when they are in the banana branch of their CDs (e.g. van der Klis 2000)

In this work we present a spectral analysis of GX 9+1 in the 0.12-18 keV energy band from a long BeppoSAX observation. We show that ionized matter is present around the system implying a relatively low value of the interstellar absorption to the source. This, in turn, suggests that the distance to the source is $\sim 5 \mathrm{kpc}$ implying that GX 9+1 has a sub-Eddington luminosity.

\section{Observation}

The BeppoSAX observation of GX 9+1 was carried out from September 16, 2001, 02:01:30.0 (UTC) to September 20, 2001, 03:00:08.5 (UTC), using the four co-aligned Narrow Field Instruments (NFIs). These are: a Low Energy Concentrator Spectrometer (LECS; 0.1-10 keV energy range; Parmar et al. 1997), two Medium Energy Concentrator Spectrometers (MECS; 1-10 keV energy range; Boella et al. 1997), a High Pressure Gas Scintillation Proportional Counter (HPGSPC; 7-60 keV energy range; Manzo et al. 1997), and a Phoswich Detector System (PDS; 13-200 keV energy range; Frontera et al. 1997). The exposure times were $\sim 60 \mathrm{ks}, \sim 149 \mathrm{ks}$, $\sim 142 \mathrm{ks}, \sim 71 \mathrm{ks}$, for LECS, MECS, HPGSPC, and PDS, respectively. The circular fields of view (FOV) of LECS and MECS are $37^{\prime}$ and $56^{\prime}$ in diameter, respectively; while those of HPGSPC and PDS are hexagonal with $F W H M$ of $78^{\prime}$ and $66^{\prime}$, respectively. The LECS and MECS detectors are position sensitive counters with imaging capability. The HPGSPC and PDS systems do not have imaging capabilities, and their data are therefore difficult to interpret and analyse for individual sources when the FOV includes more than one source. Indeed, a faint X-ray pulsar, designated SAX J1802.7-2017, was vis-

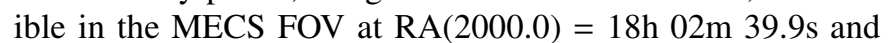
$\operatorname{Dec}(2000.0)=-20^{\circ} 17^{\prime} 13.50^{\prime \prime}$ (position uncertainty $2^{\prime}$ ), at an angular distance from GX 9+1 of 22' (Augello et al. 2003). Therefore SAX J1802.7-2017 was within the HPGSPC and PDS FOVs; the presence of the pulsar in the PDS FOV is confirmed by the detection of the X-ray pulsations in the PDS data (see Fig. 6 in Augello et al. 2003). The pulse fraction was 7\% \pm $2 \%$ and $36 \% \pm 3 \%$ in the $13-25 \mathrm{keV}$ and $25-80 \mathrm{keV}$ energy

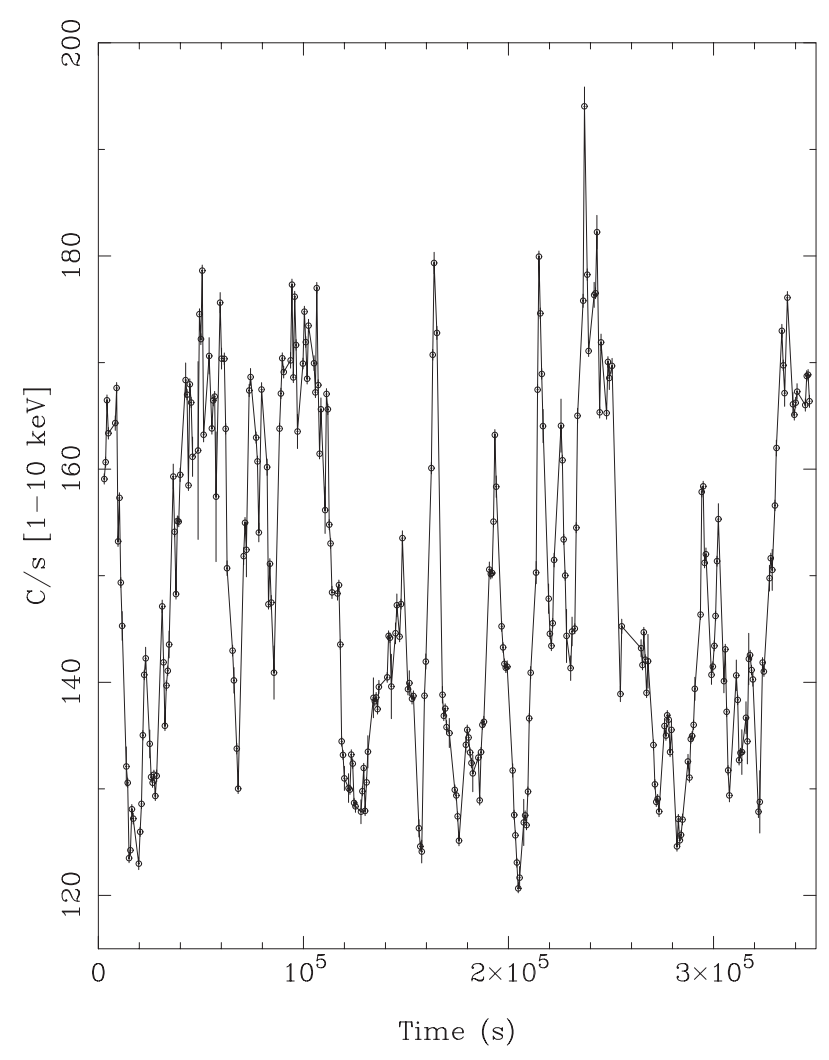

Fig. 1. GX 9+1 lightcurve in the energy band 1-10 keV (MECS data). The open circles are the GX $9+1$ count rate; for sake of clarity we interpolated them. The bin time is $697 \mathrm{~s}$.

bands, respectively (Augello et al. 2003). The lower pulse fraction in the $13-25 \mathrm{keV}$ energy band was probably due to the presence of X-ray emission from GX 9+1, which decreased markedly above $25 \mathrm{keV}$. In consideration of these results we will not use the PDS data. On the contrary, we will use the HPGSPC data in the 7-18 keV energy range, where the emission of GX 9+1 overwhelmed that of SAX J1802.7-2017, as discussed in detail below.

In Fig. 1 we show the $697 \mathrm{~s}$ binned lightcurve of GX 9+1 in the $1-10 \mathrm{keV}$ energy band. The count rate was highly variable during the entire observation ranging from $\sim 120$ counts $\mathrm{s}^{-1}$ up to $\sim 200$ counts $s^{-1}$. In Fig. 2 we show the $C D$, where the hard color $(\mathrm{HC})$ is the ratio between the count rate in the $7-10 \mathrm{keV}$ energy band to that in the $3-7 \mathrm{keV}$ energy band and the soft color (SC) is the ratio between the count rate in the 3-7 keV energy band to the count rate in the 1-3 keV energy band. Because of the high variability of GX 9+1, we selected six zones on the CD (see Fig. 2) and extracted the corresponding energy spectra from each zone. In Table 1 we report the intervals of SC and HC that identified the six selected zones; the exposure times of the LECS, MECS, and HPGSPC for each of the six intervals are also reported.

\section{Spectral analysis}

The PHA spectra of the LECS, MECS, and HPGSPC were rebinned in order to have at least 25 counts/energy channel and to oversample the instrumental energy resolution by 


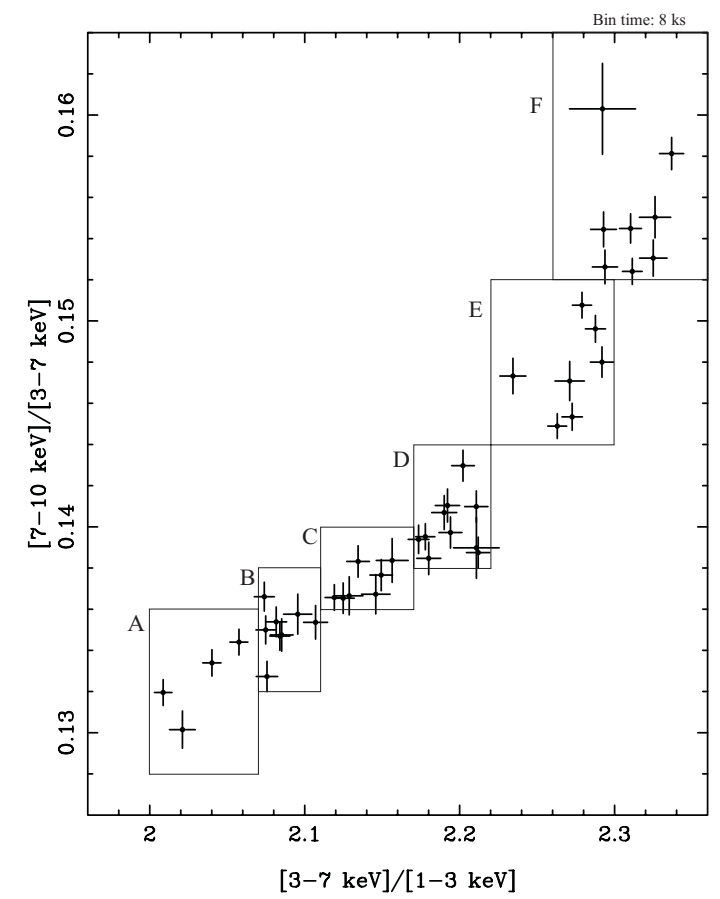

Fig. 2. Color-color diagram of GX 9+1. The hard color is the hardness ratio $[7-10 \mathrm{keV}] /[3-7 \mathrm{keV}]$ and the soft color is the hardness ratio [3-7 keV]/[1-3 keV]. The bin time is $8 \mathrm{ks}$. The boxes indicate the six selected zones from which we extracted the corresponding energy spectra.

approximately the same factor at all energies ${ }^{1}$. To account for calibration uncertainties, a systematic error of $1 \%$ was added to the data. As customary, in the spectral fitting procedure we allowed for a different normalization of the LECS and HPGSPC spectra relative to the MECS spectrum, and checked that the values obtained in the fits were in the standard range of each instrument. The energy ranges used for the spectral analysis were $0.12-2.7 \mathrm{keV}$ for the LECS, $1.8-10 \mathrm{keV}$ for the MECS, and 7-18 keV for the HPGSPC. We indicated the six spectra, corresponding to the six intervals selected in the CD (see Fig. 2), with letters from A to F.

Initially we fitted the continuum emission using a blackbody plus the Comptonization model Comptt (Titarchuk 1994) including absorption by neutral matter. This model usually gives a good fit to the spectrum of bright LMXBs (Iaria et al. 2004, and references therein). In our case we obtained an $\chi^{2} /$ d.o.f. of $201 / 167,219 / 166,166 / 167,225 / 167,177 / 167$, and $195 / 167$, respectively, for spectra $\mathrm{A}$ to $\mathrm{F}$. The equivalent hydrogen column density was $\sim 1.4 \times 10^{22} \mathrm{~cm}^{-2}$, while the blackbody temperature, the seed-photon temperature, and the electron temperature of the Comptonized component were $0.6 \mathrm{keV}, 1 \mathrm{keV}$, and $2.3 \mathrm{keV}$, respectively. The optical depth $\tau$ of the Comptonizing cloud was between 14 and 17 for a spherical geometry. The residuals with respect to this model are shown in Fig. 3 and the parameters of the fit are reported in Table 2.

The residuals showed a soft excess below $1 \mathrm{keV}$ with respect to this model which was less evident in Spectrum A

\footnotetext{
1 see the BeppoSAX cookbook at http://www.sdc.asi.it/software/index.html
}

Table 1. Some details of the BeppoSAX observation of GX 9+1. In Cols. 1 and 2 we report the intervals of Soft Color (SC) and Hard Color (HC) which identify the six selected zones in the color-color diagram (see Fig. 2). In the other columns the corresponding exposure times, in ks, of the LECS, MECS, and HPGSPC observations, respectively, are also shown.

\begin{tabular}{lccccc}
\hline \hline Zone & $\begin{array}{c}\text { SC } \\
\text { interval }\end{array}$ & $\begin{array}{c}\text { HC } \\
\text { interval }\end{array}$ & $\begin{array}{c}\text { LECS } \\
\mathrm{ks}\end{array}$ & $\begin{array}{c}\text { MECS } \\
\mathrm{ks}\end{array}$ & $\begin{array}{c}\text { HPGSPC } \\
\mathrm{ks}\end{array}$ \\
\hline $\mathrm{A}$ & $2.00-2.07$ & $0.128-0.136$ & 7 & 15 & 16 \\
$\mathrm{~B}$ & $2.07-2.11$ & $0.132-0.138$ & 11 & 30 & 30 \\
$\mathrm{C}$ & $2.11-2.17$ & $0.136-0.140$ & 11 & 24 & 22 \\
$\mathrm{D}$ & $2.17-2.22$ & $0.138-0.144$ & 15 & 32 & 29 \\
$\mathrm{E}$ & $2.22-2.30$ & $0.144-0.152$ & 10 & 23 & 21 \\
$\mathrm{~F}$ & $2.26-2.36$ & $0.152-0.164$ & 9 & 24 & 23 \\
\hline
\end{tabular}

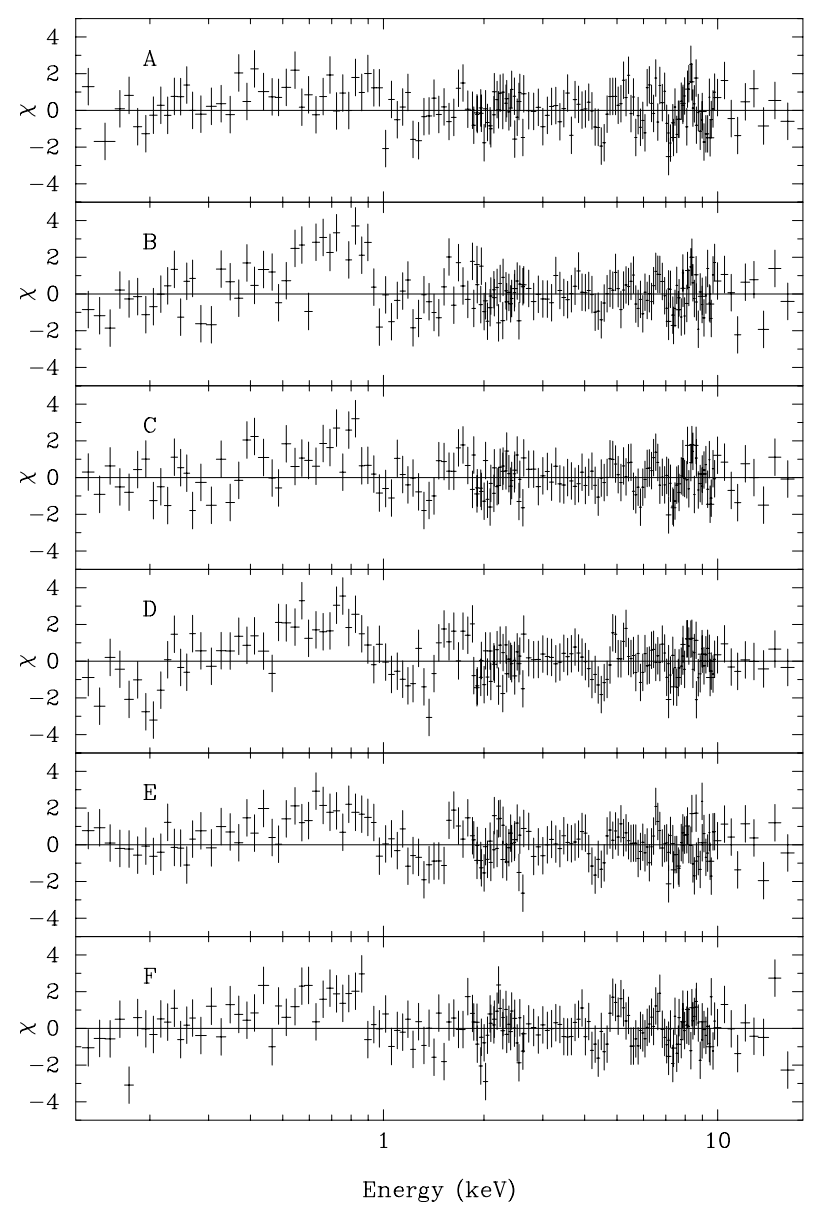

Fig. 3. Residuals in units of $\sigma$ for spectra A, B, C, D, E, and F from top to bottom, respectively. The residuals concern the model composed of a blackbody plus a Comptonized component absorbed by an equivalent hydrogen column of $1.4 \times 10^{22} \mathrm{~cm}^{-2}$ (see Table 2).

because of the lower exposure time of the LECS in this interval (see below). The excess had the same behaviour in all spectra, reaching the maximum at $0.6-0.7 \mathrm{keV}$. We tried several emission components to fit the soft excess but none gave reasonable results. The addition of another blackbody component with a temperature of $\sim 0.05 \mathrm{keV}$ gave a corresponding 
Table 2. Results of the spectral fit in the $0.12-18 \mathrm{keV}$ energy band of spectra A, B, C, D, E, and F with a simple continuum model. The model is composed of a blackbody plus a Comptonized component (Comptt in XSPEC), both absorbed by neutral matter. Uncertainties are at $90 \%$ confidence level for a single parameter; upper limits are at $95 \%$ confidence level. $k T_{\mathrm{BB}}$ and $N_{\mathrm{BB}}$ are, respectively, the blackbody temperature and normalization in units of $L_{39} / D_{10}^{2}$, where $L_{39}$ is the luminosity in units of $10^{39} \mathrm{ergs} / \mathrm{s}$ and $D_{10}$ is the distance in units of $10 \mathrm{kpc}$. $k T_{0}$ is the temperature of the seed photons for the Comptonization, $k T_{\mathrm{e}}$ is the electron temperature, $\tau$ is the optical depth of the scattering cloud using a spherical geometry, $N_{\text {comptt }}$ is the normalization of the Comptt model in XSPEC v.11.2.0 units.

\begin{tabular}{lcccccc}
\hline \hline Parameters & $\mathrm{A}$ & $\mathrm{B}$ & $\mathrm{C}$ & $\mathrm{D}$ & $\mathrm{E}$ & $\mathrm{F}$ \\
\hline$N_{\mathrm{H}}\left(\times 10^{22} \mathrm{~cm}^{-2}\right)$ & $1.369_{-0.034}^{+0.038}$ & $1.385_{-0.030}^{+0.032}$ & $1.372_{-0.033}^{+0.036}$ & $1.404_{-0.031}^{+0.035}$ & $1.375_{-0.032}^{+0.036}$ & $1.447_{-0.038}^{+0.043}$ \\
& & & & & & \\
$k T_{\mathrm{BB}}(\mathrm{keV})$ & $0.601_{-0.048}^{+0.041}$ & $0.599 \pm 0.035$ & $0.576_{-0.040}^{+0.037}$ & $0.557_{-0.036}^{+0.034}$ & $0.591_{-0.046}^{+0.041}$ & $0.546_{-0.044}^{+0.041}$ \\
$N_{\mathrm{BB}}\left(\times 10^{-2}\right)$ & $3.98_{-0.78}^{+0.70}$ & $4.20_{-0.53}^{+0.51}$ & $3.83 \pm 0.53$ & $3.70 \pm 0.46$ & $4.02_{-0.72}^{+0.69}$ & $3.79 \pm 0.63$ \\
& & & & & & \\
$k T_{0}(\mathrm{keV})$ & $0.99 \pm 0.11$ & $1.045_{-0.080}^{+0.080}$ & $1.010_{-0.076}^{+0.083}$ & $0.978_{-0.074}^{+0.069}$ & $0.986_{-0.089}^{+0.097}$ & $0.909_{-0.077}^{+0.082}$ \\
$k T_{\mathrm{e}}(\mathrm{keV})$ & $2.277_{-0.050}^{+0.075}$ & $2.322_{-0.054}^{+0.079}$ & $2.302_{-0.049}^{+0.069}$ & $2.260_{-0.037}^{+0.048}$ & $2.222_{-0.036}^{+0.052}$ & $2.204_{-0.024}^{+0.031}$ \\
$\tau$ & $14.49_{-1.06}^{+0.76}$ & $13.85_{-1.05}^{+0.80}$ & $14.15_{-0.95}^{+0.75}$ & $14.70_{-0.74}^{+0.61}$ & $15.93_{-0.98}^{+0.74}$ & $17.15_{-0.69}^{+0.55}$ \\
$N_{\text {comptt }}$ & $1.11 \pm 0.11$ & $1.132_{-0.088}^{+0.084}$ & $1.273_{-0.092}^{+0.088}$ & $1.362 \pm 0.077$ & $1.451_{-0.096}^{+0.100}$ & $1.618_{-0.085}^{+0.090}$ \\
& & & & & & \\
$\chi^{2}$ (d.o.f.) & $201(167)$ & $219(166)$ & $166(167)$ & $225(167)$ & $177(167)$ & $195(167)$ \\
\hline
\end{tabular}

unabsorbed luminosity of $\sim 10^{40} \mathrm{erg} / \mathrm{s}$ in the $0.1-200 \mathrm{keV}$ energy range assuming a distance to the source of $8 \mathrm{kpc}$. This result is very hard to explain when assuming that the X-ray binary system contains a neutron star. Instead of a blackbody we added a Gaussian line centered at $0.6 \mathrm{keV}$, also in this case, because of the strong absorption to the source; but the results were not acceptable because the corresponding equivalent width of the line was larger than $3 \mathrm{keV}$. Finally, we tried a partial covering component to fit the soft excess; and again in this case we did not obtain a good fit, bacause the addition of this component left the $\chi^{2} /$ d.o.f. values unchanged with respect to the initial model. This soft excess could therefore be explained in two ways: either that there was a continuum emission component below $1 \mathrm{keV}$ absorbed by a lower equivalent hydrogen column or that the equivalent hydrogen column of the whole spectrum was overestimated.

For the first hypothesis, the residuals below $1 \mathrm{keV}$ disappeared by adding a continuum emission component absorbed by a lower value of the equivalent hydrogen column $\left(N_{\mathrm{H}} \sim 4 \times\right.$ $\left.10^{20} \mathrm{~cm}^{-2}\right)$ than that required for the other components of the model $\left(\sim 1.4 \times 10^{22} \mathrm{~cm}^{-2}\right)$. However in this case the lower value of $N_{\mathrm{H}}$ was unrealistic since the emitting source should be less distant than $1 \mathrm{kpc}$. Because in the LECS and MECS FOV no contaminating source was visible, we should conclude that GX $9+1$ is closer than $1 \mathrm{kpc}$ or that another source is present with an angular distance within $30^{\prime \prime}$ (i.e. the position reconstruction uncertainty for MECS in the central area of $9^{\prime}$; see Boella et al. 1997) to GX 9+1, at a distance less then $1 \mathrm{kpc}$. Since this scenario seems to be contrived, in this paper we concentrate on the second hypothesis.

A good fit was obtained by adding two absorption edges associated to O VII and O VIII to the initial model as reported recently by Iaria et al. (2005) when studying the X-ray spectrum of Circinus X-1. In Fig. 4 we show residuals of the six spectra with respect to this model, and in Table 3 we report the

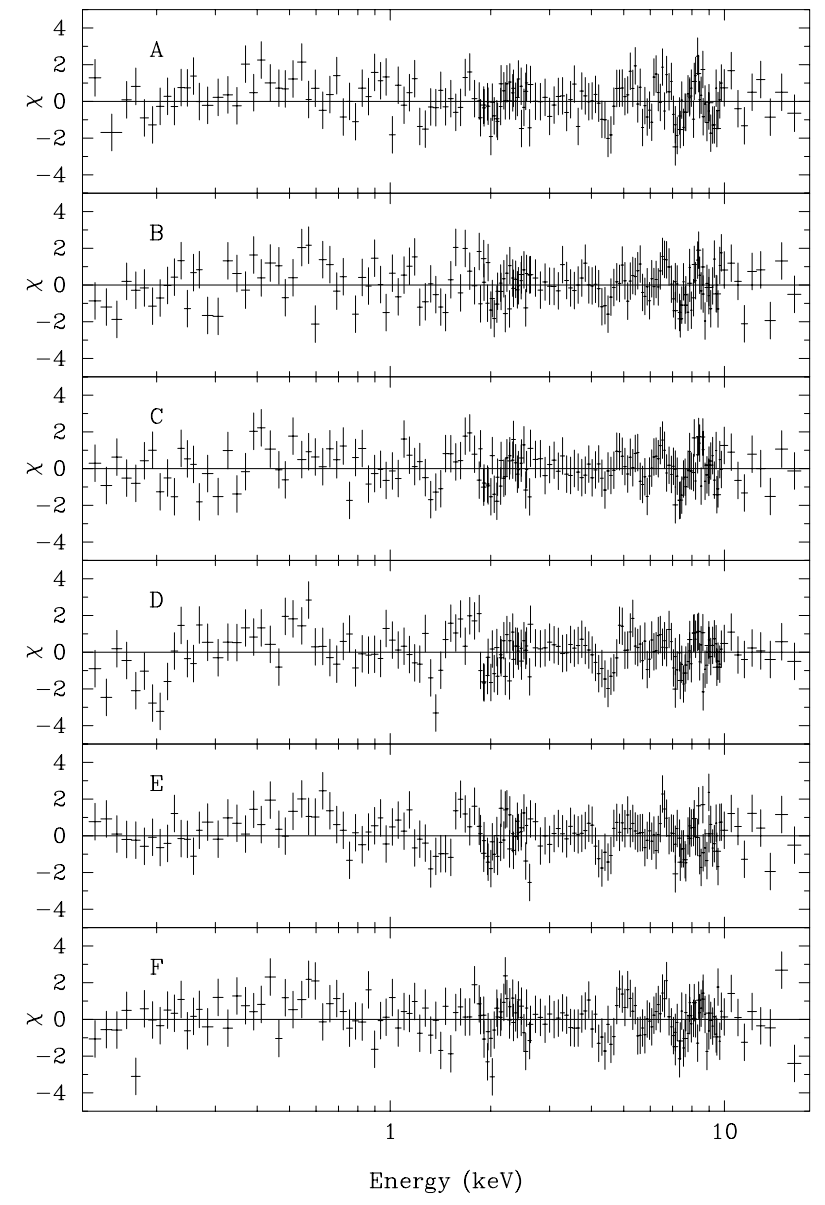

Fig. 4. Residuals in units of $\sigma$ for spectra A, B, C, D, E, and F from top to bottom, respectively. The residuals concern the model reported in Table 3.

best-fit parameters and the probability of chance improvement $f_{\mathrm{P}}$ for the addition of the O VII and O VIII absorption 
edges to the initial model. The addition of the two absorption edges allowed us to fit the energy spectrum below $1 \mathrm{keV}$. The parameters of the continuum components were unchanged with respect to the initial model, while the equivalent hydrogen column $N_{\mathrm{H}}$ became $\sim 1 \times 10^{22} \mathrm{~cm}^{-2}$. Features were still present in the residuals at $1.2 \mathrm{keV}, 4.2 \mathrm{keV}, 5.5 \mathrm{keV}$, and $7 \mathrm{keV}$. To fit these residuals we added several absorption edges by fixing the threshold energies to $1.196 \mathrm{keV}, 4.121 \mathrm{keV}, 5.470 \mathrm{keV}$, and $7.117 \mathrm{keV}$, corresponding to the absorption edges of Ne IX, Ar XVII, Ca XX, and Fe I, respectively. Near $2 \mathrm{keV}$ an absorption feature was present (see Fig. 4), which could be associated to a $L_{\alpha}$ absorption line of Si XIV. We fixed the centroid energy of the line finding the width and the intensity for the six spectra. This model provided a good fit of all our six spectra. The results are reported in Tables 4 and 5, and in Fig. 5 the residuals with respect to the best fit model are shown.

We found that the parameters of the continuum components were mostly unchanged compared to the model having only two absorption edges at low energies (Table 3 ). The equivalent hydrogen column was between $0.75 \times 10^{22} \mathrm{~cm}^{-2}$ and $0.85 \times$ $10^{22} \mathrm{~cm}^{-2}$. The blackbody temperature was $\sim 0.5 \mathrm{keV}$; the seed photon temperature $k T_{0}$ and the electron temperature $k T_{\mathrm{e}}$ of the Comptonized component were $1 \mathrm{keV}$ and $2.2 \mathrm{keV}$, respectively. The optical depths of the absorption edges associated to O VII and $\mathrm{O}$ VIII are 2 and 1.5, respectively. In the spectra A and C we found an upper limit to the optical depth associated to the O VII absorption edge, which was compatible with the value obtained in our other spectra. Since the value of $N_{\mathrm{H}}$ was similar in the six spectra we suggest that the less evident detection of the soft excess in spectrum A (see Fig. 3) was due to its lower LECS statistics (see Table 1). The optical depths associated to the Ne IX, Ar XVII, and Ca XX absorption edge were around $0.16,0.03$, and 0.03 , respectively. The width and the intensity of the $L_{\alpha}$ absorption line associated to Si XIV were less than $0.20 \mathrm{keV}$ and $\sim-5 \times 10^{-3}$ photons $\mathrm{cm}^{-2} \mathrm{~s}^{-1}$, respectively.

\section{Discussion}

We analyzed data of GX 9+1 from a BeppoSAX observation taken in September 2001. The lightcurve showed high variability of the source during the observation. We selected six regions in the $\mathrm{CD}$ of the source and extracted from each interval the corresponding spectrum. Because of the presence of the X-ray pulsar SAX J1802.7-2017 in the HPGSPC and PDS FOV (Augello et al. 2003), we did not include the PDS data in our spectral analysis, while we used the HPGSPC data up to $18 \mathrm{keV}$ under the hypothesis that in this energy range the emission of GX 9+1 overwhelmed that of SAX J1802.7-2017. The model used to fit the spectrum of SAX J1802.7-2017 was a power-law component with a photon index of -0.1 and a normalization of $1.05 \times 10^{-3}$ absorbed by an equivalent hydrogen column of $1.7 \times 10^{22} \mathrm{~cm}^{-2}$ (Augello et al. 2003). Extrapolating this spectrum at higher energies, the corresponding flux of the pulsar, in the energy range $10-18 \mathrm{keV}$, was $\sim 2.4 \times 10^{-10} \mathrm{erg} \mathrm{cm}^{-2} \mathrm{~s}^{-1}$. On the other hand, the flux of GX $9+1$ in the energy range $10-18 \mathrm{keV}$, using the model reported in Tables 4 and 5, varied between $1.9 \times$ $10^{-9} \mathrm{erg} \mathrm{cm}^{-2} \mathrm{~s}^{-1}$ and $3.0 \times 10^{-9} \mathrm{erg} \mathrm{cm}^{-2} \mathrm{~s}^{-1}$ going from

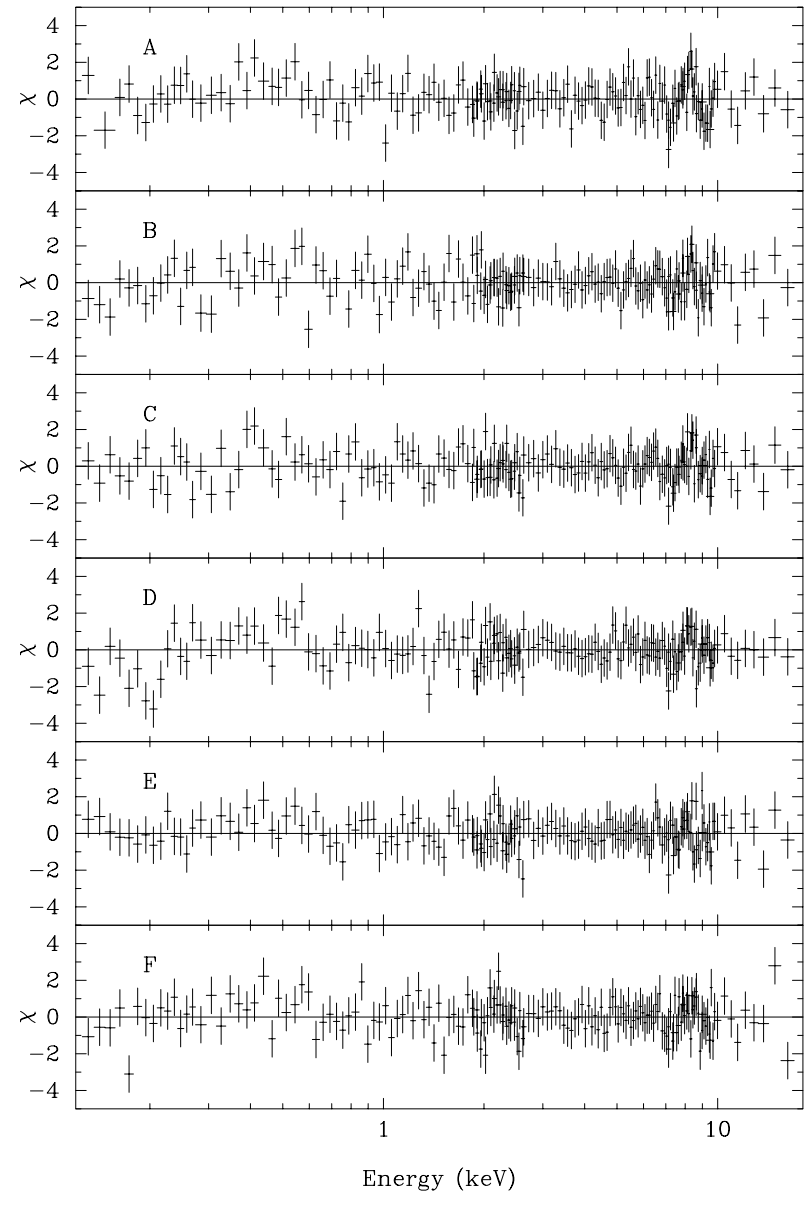

Fig. 5. Residuals in units of $\sigma$ for spectra A, B, C, D, E, and F from top to bottom, respectively. The residuals concern the final model reported in Tables 4 and 5.

Spectrum A to Spectrum F. This implied that, in this energy band, the flux associated to GX 9+1 was a factor 10 larger than that of the contaminating source SAX J1802.7-2017. Note that the adopted model for the spectrum of SAX J1802.7-2017 was unrealistic for energies larger than $10 \mathrm{keV}$ because the continuum emission of X-ray pulsars is generally fitted by a power-law component that has a cut-off at energies higher than $\sim 10 \mathrm{keV}$; the cut-off might further reduce the flux of the pulsar in the hard X-ray range. These results led us to conclude that the influence of the pulsar emission in the 10-18 keV energy band was negligible. Consequently we studied the six energy spectra of GX $9+1$ in the energy band $0.12-18 \mathrm{keV}$.

The spectra were initially fitted to a blackbody plus a Comptonized component absorbed by an equivalent hydrogen column of $\sim 1.4 \times 10^{22} \mathrm{~cm}^{-2}$; however, below $1 \mathrm{keV}$ a soft excess was present in the residuals of this model. We obtained a good fit using the same components described above for the continuum emission, with the addition of the absorption edges associated to O VII, O VIII, Ne IX, Ar XVII, Ca XX, and Fe I, and an absorption line associated to Si XIV (see below). A similar model was used to fit the spectrum of the bright LMXB Cir X-1 (Iaria et al. 2004). The final model gave a value of the equivalent hydrogen column associated to the interstellar absorption 
Table 3. Results of the spectral fit in the $0.12-18 \mathrm{keV}$ energy band of spectra A, B, C, D, E, and F. The model is the same reported in Table 2 with the addition of the absorption edges associated to O VII and O VIII. $\tau_{\mathrm{O} \text { VII }}$ and $\tau_{\mathrm{O} \text { VIII }}$ indicate the optical depth associated to the $\mathrm{O}$ VII and $\mathrm{O}$ VIII absorption edges, respectively. $f_{\mathrm{P}}$ is the probability of chance improvement for the addition of the O VII and O VIII absorption edges.

\begin{tabular}{|c|c|c|c|c|c|c|}
\hline Parameters & A & B & $\mathrm{C}$ & $\bar{D}$ & $\bar{E}$ & $\bar{F}$ \\
\hline$N_{\mathrm{H}}\left(\times 10^{22} \mathrm{~cm}^{-2}\right)$ & $1.10_{-0.16}^{+0.19}$ & $0.74_{-0.11}^{+0.18}$ & $1.012_{-0.189}^{+0.084}$ & $0.79_{-0.11}^{+0.26}$ & $0.994_{-0.333}^{+0.051}$ & $1.039_{-0.203}^{+0.056}$ \\
\hline$E_{\mathrm{O} \text { VII }}(\mathrm{keV})$ & 0.739 (fixed) & 0.739 (fixed) & 0.739 (fixed) & 0.739 (fixed) & 0.739 (fixed) & 0.739 (fixed) \\
\hline$\tau_{\mathrm{O} \text { VII }}$ & $<0.78$ & $<1.73$ & $<1.05$ & $<2.13$ & $<2.09$ & $<1.08$ \\
\hline$E_{\mathrm{O} \text { VIII }}(\mathrm{keV})$ & 0.871 (fixed) & 0.871 (fixed) & 0.871 (fixed) & 0.871 (fixed) & 0.871 (fixed) & 0.871 (fixed) \\
\hline$\tau_{\mathrm{O} \text { VIII }}$ & $1.03_{-0.72}^{+0.41}$ & $1.88_{-0.26}^{+0.21}$ & $1.38_{-0.31}^{+0.26}$ & $1.55_{-0.29}^{+0.22}$ & $1.45_{-0.32}^{+0.26}$ & $1.55_{-0.34}^{+0.28}$ \\
\hline$k T_{\mathrm{BB}}(\mathrm{keV})$ & $0.605_{-0.053}^{+0.045}$ & $0.606_{-0.045}^{+0.041}$ & $0.573_{-0.023}^{+0.034}$ & $0.558 \pm 0.043$ & $0.590_{-0.050}^{+0.023}$ & $0.544_{-0.024}^{+0.039}$ \\
\hline$N_{\mathrm{BB}}\left(\times 10^{-2}\right)$ & $3.71_{-0.80}^{+0.077}$ & $3.53 \pm 0.61$ & $3.39_{-0.54}^{+0.58}$ & $3.01_{-0.47}^{+0.61}$ & $3.53 \pm 0.75$ & $3.29 \pm 0.63$ \\
\hline$k T_{0}(\mathrm{keV})$ & $0.99 \pm 0.11$ & $1.029_{-0.081}^{+0.090}$ & $0.996_{-0.068}^{+0.078}$ & $0.969_{-0.062}^{+0.069}$ & $0.973_{-0.080}^{+0.091}$ & $0.904_{-0.067}^{+0.073}$ \\
\hline$k T_{\mathrm{e}}(\mathrm{keV})$ & $2.279_{-0.049}^{+0.073}$ & $2.324_{-0.051}^{+0.073}$ & $2.302_{-0.044}^{+0.058}$ & $2.267_{-0.036}^{+0.046}$ & $2.225_{-0.029}^{+0.042}$ & $2.210_{-0.018}^{+0.026}$ \\
\hline$\tau$ & $14.49_{-1.02}^{+0.73}$ & $13.91_{-0.95}^{+0.73}$ & $14.18_{-0.88}^{+0.61}$ & $14.64_{-0.69}^{+0.57}$ & $15.92_{-0.81}^{+0.29}$ & $17.06_{-0.57}^{+0.44}$ \\
\hline$N_{\text {comptt }}$ & $1.11 \pm 0.11$ & $1.133_{-0.092}^{+0.089}$ & $1.281 \pm 0.045$ & $1.359 \pm 0.079$ & $1.456 \pm 0.092$ & $1.615 \pm 0.079$ \\
\hline$f_{\mathrm{P}}$ & 0.19 & $1.5 \times 10^{-9}$ & $7.7 \times 10^{-5}$ & $3.7 \times 10^{-7}$ & $1.0 \times 10^{-9}$ & $1.0 \times 10^{-3}$ \\
\hline$\chi^{2}$ (d.o.f.) & $197(165)$ & $171(164)$ & $148(165)$ & $188(165)$ & $159(165)$ & $180(165)$ \\
\hline
\end{tabular}

Table 4. Parameters of the continuum model that gives the best fit in the $0.12-18 \mathrm{keV}$ energy band for spectra A, B, C, D, E, and F. The model is composed by a blackbody plus a Comptonized component including absorption by neutral matter. Uncertainties are at $90 \%$ confidence level for a single parameter; upper limits are at $95 \%$ confidence level. $R_{\mathrm{BB}}$ is the blackbody radius, $R_{W}$ is the seed-photon radius, and $L_{\mathrm{tot}}$ is the unabsorbed luminosity of the source in the energy range $0.12-18 \mathrm{keV} . R_{\mathrm{BB}}, R_{W}$, and $L_{\mathrm{tot}}$ are calculated assuming a distance to the source of

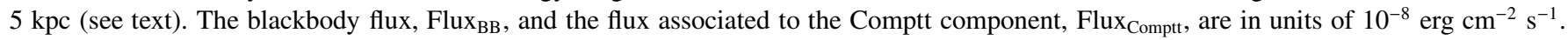
The other parameters are defined as in Tables 2 and 3. $f_{\mathrm{P}}$ is the probability of chance improvement for the addition of the Ne IX, Ar XVII, Ca XX and Fe I absorption edges, and of the Si XIV absorption line to the model reported in Table 3.

\begin{tabular}{|c|c|c|c|c|c|c|}
\hline Continuum & $\bar{A}$ & $\bar{B}$ & $\mathrm{C}$ & $\bar{D}$ & $\bar{E}$ & $\bar{F}$ \\
\hline$N_{\mathrm{H}}\left(\times 10^{22} \mathrm{~cm}^{-2}\right)$ & $0.92_{-0.18}^{+0.32}$ & $0.718_{-0.079}^{+0.125}$ & $0.82_{-0.11}^{+0.26}$ & $0.793_{-0.080}^{+0.120}$ & $0.728_{-0.044}^{+0.067}$ & $0.771_{-0.055}^{+0.187}$ \\
\hline$k T_{\mathrm{BB}}(\mathrm{keV})$ & $0.534_{-0.061}^{+0.068}$ & $0.531_{-0.064}^{+0.076}$ & $0.544_{-0.066}^{+0.072}$ & $0.471_{-0.038}^{+0.047}$ & $0.490_{-0.055}^{+0.057}$ & $0.517_{-0.050}^{+0.052}$ \\
\hline$N_{\mathrm{BB}}\left(\times 10^{-2}\right)$ & $3.54_{-0.61}^{+0.701}$ & $3.34_{-0.50}^{+0.604}$ & $3.71_{-0.63}^{+0.33}$ & $3.48_{-0.43}^{+0.52}$ & $3.40_{-0.33}^{+0.00}$ & $3.91 \pm 0.48$ \\
\hline Flux $_{\mathrm{BB}}$ & $\sim 0.30$ & $\sim 0.28$ & $\sim 0.31$ & $\sim 0.29$ & $\sim 0.28$ & $\sim 0.33$ \\
\hline$R_{\mathrm{BB}}(\mathrm{km})$ & $29 \pm 10$ & $29 \pm 11$ & $29 \pm 10$ & $37 \pm 10$ & $34 \pm 11$ & $33 \pm 9$ \\
\hline$k T_{0}(\mathrm{keV})$ & $0.99_{-0.10}^{+0.13}$ & $1.003_{-0.097}^{+0.132}$ & $1.05_{-0.11}^{+0.14}$ & $0.968_{-0.065}^{+0.071}$ & $0.958_{-0.038}^{+0.088}$ & $0.998_{-0.092}^{+0.099}$ \\
\hline$k T_{\mathrm{e}}(\mathrm{keV})$ & $2.290_{-0.057}^{+0.10}$ & $2.318_{-0.054}^{+0.095}$ & $2.331_{-0.069}^{+0.11}$ & $2.271_{-0.041}^{-0.065}$ & $2.226 \pm 0.022$ & $2.225_{-0.038}^{-0.092}$ \\
\hline$\tau$ & $13.91_{-1.18}^{+0.091}$ & $13.68_{-1.26}^{+0.84}$ & $13.5_{-1.6}^{+0.1 .1}$ & $14.24_{-0.81}^{+0.071}$ & $15.47_{-1.02}^{+0.83}$ & $16.16_{-1.10}^{+0.96}$ \\
\hline$N_{\text {comptt }}$ & $1.18_{-0.13}^{+0.10}$ & $1.22_{-0.14}^{+0.10}$ & $1.28_{-0.15}^{+0.11}$ & $1.438_{-0.064}^{+0.073}$ & $1.563_{-0.059}^{+0.079}$ & $1.620_{-0.085}^{+0.1077}$ \\
\hline Flux $_{\text {Comptt }}$ & $\sim 1.32$ & $\sim 1.38$ & $\sim 1.47$ & $\sim 1.59$ & $\sim 1.73$ & $\sim 1.85$ \\
\hline$R_{W}(\mathrm{~km})$ & $8 \pm 2$ & $8 \pm 2$ & $8 \pm 2$ & $9 \pm 1$ & $9 \pm 2$ & $9 \pm 2$ \\
\hline$L_{\text {tot }}\left(\times 10^{37} \mathrm{erg} \mathrm{s}^{-1}\right)$ & $\sim 4.8$ & $\sim 4.9$ & $\sim 5.2$ & $\sim 5.5$ & $\sim 5.9$ & $\sim 6.4$ \\
\hline$f_{\mathrm{P}}$ & $3.3 \times 10^{-3}$ & $3.4 \times 10^{-4}$ & $4.3 \times 10^{-5}$ & $5.5 \times 10^{-7}$ & $2.2 \times 10^{-8}$ & $2.9 \times 10^{-6}$ \\
\hline$\chi^{2}$ (d.o.f.) & 174 (159) & 148 (159) & 123 (159) & 147 (159) & 119 (159) & 144 (159) \\
\hline
\end{tabular}

between $\sim 0.7 \times 10^{22} \mathrm{~cm}^{-2}$ and $\sim 0.9 \times 10^{22} \mathrm{~cm}^{-2}$, which was a factor two smaller than in the initial model.

The distance to GX 9+1 is not known but an upper limit was assumed to be at the distance of the Galactic Center $(10 \mathrm{kpc}$,
White et al. 1988). The absorbing equivalent hydrogen column density estimated by White et al. (1988) and Predehl \& Schmitt (1995) was $N_{\mathrm{H}} \sim 1.5 \times 10^{22} \mathrm{~cm}^{-2}$. First of all we estimated a new value of the distance to the source based on this value 
Table 5. Parameters of the absorption edges and the absorption line included in the best fit model of spectra A, B, C, D, E, and F. The Uncertainties are at $90 \%$ confidence level for a single parameter; upper limits are at $95 \%$ confidence level. E and $\tau$ indicate the energy and optical depths of the absorption edges. $E_{\text {line }}, \sigma_{\text {line }}, I_{\text {line }}$, and $E Q W_{\text {Si XIV }}$ are the centroid, the width, the intensity, and the equivalent width of the absorption line. $I_{\text {line }}$ is in units of photons $\mathrm{cm}^{-2} \mathrm{~s}^{-1} . r$ and $n_{\mathrm{e}}$ indicate the distance to the source where the line is produced and the corresponding electron density (see text).

\begin{tabular}{|c|c|c|c|c|c|c|}
\hline$\overline{\text { Features }}$ & $\overline{\mathrm{A}}$ & $\overline{\mathrm{B}}$ & $\overline{\overline{\mathrm{C}}}$ & $\overline{\mathrm{D}}$ & $\overline{\mathrm{E}}$ & $\overline{\overline{\mathrm{F}}}$ \\
\hline$E_{\mathrm{O} \text { VII }}(\mathrm{keV})$ & 0.739 (fixed) & 0.739 (fixed) & 0.739 (fixed) & 0.739 (fixed) & 0.739 (fixed) & 0.739 (fixed) \\
\hline$\tau_{\mathrm{O} \text { VII }}$ & $<3.0$ & $1.43_{-0.85}^{+0.72}$ & $<2.5$ & $1.90_{-0.86}^{+0.79}$ & $2.40_{-0.99}^{+0.36}$ & $2.0_{-1.3}^{+1.1}$ \\
\hline$E_{\mathrm{O} \text { VIII }}(\mathrm{keV})$ & 0.871 (fixed) & 0.871 (fixed) & 0.871 (fixed) & 0.871 (fixed) & 0.871 (fixed) & 0.871 (fixed) \\
\hline$\tau_{\mathrm{O} \text { VIII }}$ & $1.15_{-0.68}^{+0.39}$ & $1.88_{-0.32}^{+0.26}$ & $1.43_{-0.41}^{+0.30}$ & $1.60_{-0.36}^{+0.27}$ & $1.30_{-0.45}^{+0.41}$ & $1.50_{-0.54}^{+0.36}$ \\
\hline$E_{\text {Ne IX }}(\mathrm{keV})$ & 1.196 (fixed) & 1.196 (fixed) & 1.196 (fixed) & 1.196 (fixed) & 1.196 (fixed) & 1.196 (fixed) \\
\hline$\tau_{\text {Ne IX }}$ & $0.16 \pm 0.10$ & $0.116_{-0.089}^{+0.093}$ & $0.119_{-0.082}^{+0.099}$ & $0.193_{-0.089}^{+0.093}$ & $0.237_{-0.089}^{+0.024}$ & $0.166_{-0.086}^{+0.091}$ \\
\hline$E_{\text {Ar XVII }}(\mathrm{keV})$ & 4.121 (fixed) & 4.121 (fixed) & 4.121 (fixed) & 4.121 (fixed) & 4.121 (fixed) & 4.121 (fixed) \\
\hline$\tau_{\mathrm{Ar} \mathrm{XVII}}$ & $0.034 \pm 0.019$ & $0.031 \pm 0.017$ & $<0.034$ & $0.038 \pm 0.015$ & $0.037 \pm 0.016$ & $0.033 \pm 0.016$ \\
\hline$E_{\mathrm{Ca} X \mathrm{XX}}(\mathrm{keV})$ & 5.470 (fixed) & 5.470 (fixed) & 5.470 (fixed) & 5.470 (fixed) & 5.470 (fixed) & 5.470 (fixed) \\
\hline$\tau_{\mathrm{CaXX}}$ & $0.032 \pm 0.020$ & $0.022 \pm 0.018$ & $0.029 \pm 0.019$ & $0.025 \pm 0.018$ & $0.0271_{-0.0061}^{+0.0180}$ & $0.039 \pm 0.017$ \\
\hline$E_{\mathrm{Fe} \mathrm{I}}(\mathrm{keV})$ & 7.117 (fixed) & 7.117 (fixed) & 7.117 (fixed) & 7.117 (fixed) & 7.117 (fixed) & 7.117 (fixed) \\
\hline$\tau_{\mathrm{Fe} \mathrm{I}}$ & $0.029 \pm 0.019$ & $0.021 \pm 0.016$ & $0.026 \pm 0.017$ & $0.021 \pm 0.014$ & $0.0244_{-0.0057}^{+0.0138}$ & $0.034 \pm 0.014$ \\
\hline$E_{\mathrm{Si} \text { XIV }}(\mathrm{keV})$ & 2.006 (fixed) & 2.006 (fixed) & 2.006 (fixed) & 2.006 (fixed) & 2.006 (fixed) & 2.006 (fixed) \\
\hline$\sigma_{\mathrm{Si} \text { XIV }}(\mathrm{keV})$ & $<0.15$ & 0.05 (fixed) & $<0.16$ & $<0.26$ & $<0.12$ & $<0.06$ \\
\hline$I_{\mathrm{Si} \text { XIV }}\left(\times 10^{-3}\right)$ & $-5.8_{-4.9}^{+3.1}$ & $-5.1 \pm 2.9$ & $-7.0_{-6.3}^{+3.3}$ & $-10.8_{-14.9}^{+5.7}$ & $-7.2_{-3.0}^{+1.5}$ & $-6.7_{-3.2}^{+2.9}$ \\
\hline$E Q W_{\mathrm{Si} X I V}(\mathrm{eV})$ & $\sim 8^{-4.9}$ & $\sim 7$ & $\sim 10$ & $\sim 14^{-14.9}$ & $\sim 9^{-3.0}$ & $\sim 8^{-3.2}$ \\
\hline$r\left(\times 10^{11}\right)(\mathrm{cm})$ & $2.3_{-1.1}^{+2.6}$ & $2.7_{-1.0}^{+3.6}$ & $2.2_{-1.1}^{+2.0}$ & $1.61_{-0.93}^{+1.80}$ & $2.77_{-0.82}^{+0.73}$ & $3.5_{-1.1}^{+2.7}$ \\
\hline$n_{\mathrm{e}}\left(\times 10^{12}\right)\left(\mathrm{cm}^{-3}\right)$ & $4.7_{-3.7}^{-11.2}$ & $3.4_{-2.8}^{+5.0}$ & $5.4_{-3.9}^{+14.0}$ & $10.8_{-8.4}^{+50.5}$ & $3.9_{-1.5}^{+3.9}$ & $\begin{array}{l}2.7_{-1.8}^{+3.2} \\
\end{array}$ \\
\hline
\end{tabular}

of $N_{\mathrm{H}}$, using the code developed by Taylor \& Cordes (1993) to map the ionized hydrogen of our Galaxy. Assuming that the measured $N_{\mathrm{H}}$ is totally associated to the interstellar matter and that the $10 \%$ of the interstellar hydrogen is ionized, we found that the corresponding dispersion measure DM associated to this $N_{\mathrm{H}}$ is $\sim 455 \mathrm{pc} \mathrm{cm}^{-3}$. Knowing that the Galactic coordinates of GX $9+1$ are $l=9.072^{\circ}$ and $b=1.172^{\circ}$, a value of $\mathrm{DM} \sim 455 \mathrm{pc} \mathrm{cm}^{-3}$ in the direction of GX $9+1$ gives a distance to the source of $d=6.4 \pm 1.9 \mathrm{kpc}$. In our final model, $N_{\mathrm{H}}$ was $\sim 0.8 \times 10^{22} \mathrm{~cm}^{-2}$, which gives a DM $\sim 260 \mathrm{pc} \mathrm{cm}^{-3}$ and a corresponding distance to the source of $d=4.4 \pm 1.3 \mathrm{kpc}$. In the following we assume $5 \mathrm{kpc}$ as distance to the source, which is compatible with the distances derived from both the $N_{\mathrm{H}}$ values discussed above. Our estimation of the distance to GX 9+1 implies that the source has a luminosity of $\sim 6 \times 10^{37} \mathrm{erg} / \mathrm{s}$ (in the 0.1-18 keV energy range) and not near the Eddington luminosity as supposed until now.

As already mentioned, the model used to fit the continuum emission is composed of a blackbody plus a Comptonized component. The corresponding radius of the blackbody component, assuming a spherical emission, was $\sim 30 \mathrm{~km}$. This radius is too large to be associated with the emission from the neutron star surface; therefore the blackbody emission was probably produced by the inner part of the accretion disk. Following in 't Zand et al. (1999) we calculated the radius $R_{W}$ of the seed-photon emitting region using the parameters reported in
Table 4 and obtained $R_{W} \sim 10 \mathrm{~km}$, which implies that the seed photons for the Comptonization may come from the neutron star surface. The parameters associated to the blackbody did not vary significantly going from spectrum A to F. The flux of the Comptonized component increased going from $\sim 1.3 \times$ $10^{-8} \mathrm{erg} \mathrm{cm}^{-2} \mathrm{~s}^{-1}$ (spectrum A) to $\sim 1.9 \times 10^{-8} \mathrm{erg} \mathrm{cm}^{-2} \mathrm{~s}^{-1}$ (spectrum F), while the other parameters associated to the Comptonized component did not vary significantly. Finally, we used the relations reported by Titarchuk \& Lyubarskij (1995) to obtain the spectral index associated to the Comptonized component (in spherical geometry) for each of the six spectra. The average spectral index was $0.80 \pm 0.15$, compatible for each spectrum.

In the six spectra a $L_{\alpha}$ absorption line associated to Si XIV was present at $2 \mathrm{keV}$. The ionization parameter $\log _{10} \xi$, corresponding to Si XIV, is 2.3. From $\xi=L_{\mathrm{x}} /\left(n_{\mathrm{e}} r^{2}\right)$ (see Krolik et al. 1981) and $L_{\text {line }}=4 \pi D^{2} I_{\text {line }}=n_{\mathrm{e}}^{2} V \alpha A f$, we could obtain both an upper limit on the distance to the central source and the corresponding electron density of the region where the line was produced. In this formula $L_{\mathrm{x}}$ is the total X-ray unabsorbed luminosity of the source in the energy band $0.12-18 \mathrm{keV}, n_{\mathrm{e}}$ the electron density, $L_{\text {line }}$ the luminosity of the line, $D$ the distance to the source, $I_{\text {line }}$ the intensity of the line, $V$ the emitting volume, $\alpha$ the recombination parameter, $A$ the cosmic abundance of the element, and $f$ the fractional number of ions of the considered element in the given ionization state. We assumed 
a spherical emitting volume $V$ of radius $r$ and $f=0.6$. The recombination parameter $\alpha$ is obtained using the relation and the best fit parameters for Si XIV reported by Verner \& Ferland (1996), where we fixed the plasma temperature at the electron temperature of the Comptonizing cloud. Using the parameters in Tables 4 and 5 we found that the line was produced at $r \sim 2 \times$ $10^{11} \mathrm{~cm}$ with a corresponding electron density of $n_{\mathrm{e}} \sim 5 \times$ $10^{12} \mathrm{~cm}^{-3}$; the values of $r$ and $n_{\mathrm{e}}$ are reported for each spectrum in Table 5.

The absorption edges associated to O VII, O VIII, and Ne IX were probably produced in a plasma with a ionization parameter $\log _{10} \xi$ of around 1.3. We obtained the equivalent hydrogen column corresponding to the optical depth of the absorption edges using $\tau_{X}=\sigma_{X} N_{\mathrm{H}} A_{X} f$, where $\sigma_{X}$ is the photoionization cross section for the ion $X, N_{\mathrm{H}}$ is the corresponding equivalent hydrogen column, $A_{X}$ the abundance of the element $X$, and $f$ the fractional number of ions of the considered element in the given ionization state. The value of the photoionization cross sections are $2.4 \times 10^{-19} \mathrm{~cm}^{2}, 9.9 \times 10^{-20} \mathrm{~cm}^{2}$, and $1.5 \times$ $10^{-19} \mathrm{~cm}^{2}$ for O VII, O VIII, and Ne IX, respectively (see Verner et al. 1996). Using the values of $\tau$ reported in Table 5, the cosmic abundance of oxygen and neon, and assuming an ionization parameter $\log _{10} \xi=1.3$ (implying $f \sim 0.5$ for $\mathrm{O}$ VII, O VIII, and $\mathrm{Ne}$ IX) we found that $N_{\mathrm{H}_{\mathrm{O}} \mathrm{VII}} \sim 2 \times 10^{22} \mathrm{~cm}^{-2}, N_{\mathrm{H}_{\mathrm{OVIII}}} \sim$ $3 \times 10^{22} \mathrm{~cm}^{-2}$ and $N_{\mathrm{H}_{\mathrm{Ne}} \mathrm{IX}} \sim 1.5 \times 10^{22} \mathrm{~cm}^{-2}$. The absorption edge associated to Ar XVII was probably produced in a plasma with an ionization parameter $\log _{10} \xi$ of around 2.9 with a corresponding $f$ of 0.7 . The value of the photoionization cross sections associated to Ar XVII is $4.2 \times 10^{-20} \mathrm{~cm}^{2}$ (Verner et al. 1996); and by assuming cosmic abundance of argon we found that $N_{\mathrm{H}_{\mathrm{Ar} \text { XVII }}}$ Was $\sim 10^{23} \mathrm{~cm}^{-2}$. Finally, the absorption edge associated to $\mathrm{Ca} \mathrm{XX}$ was probably produced in a plasma with a ionization parameter $\log _{10} \xi$ of around 3.5 with a corresponding $f$ of 0.7 . The value of the photoionization cross sections associated to Ca XX was $1.6 \times 10^{-19} \mathrm{~cm}^{2}$ (Verner et al. 1996); if assuming cosmic abundance of calcium, we found that $N_{\mathrm{H} \text { Ca XX }}$ is $\sim 10^{23} \mathrm{~cm}^{-2}$.

We could also estimate an upper limit on the distance $d$ to the emitting source of the region where the absorption edges were produced. To do it we used $d<L_{\mathrm{x}} /(N \xi)$ (Reynolds \& Fabian 1995), where $N$ is the equivalent hydrogen column associated to the photoionized matter, $d$ the distance to the central object, $L_{\mathrm{x}}$ the unabsorbed luminosity of the source in the energy range $0.12-18 \mathrm{keV}$, and $\xi$ is the ionization parameter defined above. We found that the O VII, O VIII, and Ne IX absorption edges were produced at a distance to the central object of $d<1.5 \times 10^{14} \mathrm{~cm}$, the Ar XVII absorption edge at $d<8 \times$ $10^{11} \mathrm{~cm}$, and, finally, the Ca XX absorption edge at $d<2 \times$ $10^{11} \mathrm{~cm}$.

These results indicate that ionized matter was present around the system; at $r \sim 2 \times 10^{11} \mathrm{~cm}$ the electron density of the plasma was $n_{\mathrm{e}} \sim 10^{12} \mathrm{~cm}^{-3}$ as indicated by the Si XIV absorption line. Since Si XIV and Ar XVII peak at the same value of the ionization parameter $(\log \xi=2.9)$, the region producing the Si XIV absorption line and the Ar XVII absorption edge was probably the same, which implies that at $r \sim 2 \times 10^{11} \mathrm{~cm}$ the equivalent hydrogen column associated to the ionized matter was $\sim 10^{23} \mathrm{~cm}^{-2}$. The Ca XX absorption edge was probably produced in the innermost region because of the larger value of the ionization parameter associated to $\mathrm{Ca} \mathrm{XX}$. In this region the value of the equivalent hydrogen column associated to the ionized matter remained $\sim 10^{23} \mathrm{~cm}^{-2}$. The absorption edges associated to O VII, O VIII, and Ne IX were produced at larger distances; and in this case the associated equivalent hydrogen column was one order of magnitude lower, indicating that further away from the neutron star the density of the ionized matter decreased, as expected.

Finally in the six spectra we observed the presence of an absorption edge associated to neutral iron. Knowing that the Fe I cross section is $3.37 \times 10^{-20} \mathrm{~cm}^{2}$ and assuming the cosmic abundance of iron, we found that the equivalent hydrogen column associated to neutral iron was $\sim 1.5 \times 10^{22} \mathrm{~cm}^{-2}$. The estimated equivalent hydrogen column associated to neutral iron was about 2 times larger than that seen in the low-energy (interstellar) absorption, indicating an overabundance of iron or perhaps a special geometry. A possible geometry was suggested by Singh \& Apparao (1994) who found similar results for the Atoll source 4U 0614+09 when analyzing EXOSAT data. The Fe I absorption edge is imprinted on the spectrum due to reflection by cold or partially ionized matter that is not in the same line of sight of the direct emission, therefore leading to an estimate of $N_{\mathrm{H}}$, which is different from the value obtained from low-energy absorption.

\section{Conclusions}

In this work we present the spectral analysis of GX 9+1 in the 0.12-18 keV energy band, using a $350 \mathrm{ks}$ long BeppoSAX observation. We selected six zones in the color-color diagram and extracted an energy spectrum from each zone. We find that the continuum is well-fitted using a blackbody plus a Comptonized component and infer that the blackbody emission can be associated to the accretion disk and that the Comptonized component can be explained as the emission from an optically thick spherical corona around the neutron star.

Our main results are the detection of ionized matter around the system producing several absorption edges in the energy spectrum and a possible reduction in the estimate of the distance to GX 9+1. The presence of several absorption edges and an absorption line associated to H-like and He-like ions indicate that the electron density of this plasma is $n_{\mathrm{e}} \sim 10^{12} \mathrm{~cm}^{-3}$ at $r \sim 2 \times 10^{11} \mathrm{~cm}$. The addition of these features in the six analyzed spectra gives the value of the equivalent hydrogen column density associated to interstellar absorption of $\sim 0.8 \times$ $10^{22} \mathrm{~cm}^{-2}$. Considering the position of GX 9+1, this implies a distance to the source of about $5 \mathrm{kpc}$. An important consequence of this result is that the atoll source GX $9+1$ has an unabsorbed luminosity of $\sim 6 \times 10^{37} \mathrm{erg} \mathrm{s}^{-1}$ in the energy range $0.12-200 \mathrm{keV}$; i.e. the source is not as bright as supposed up to now.

Acknowledgements. We thank M. Burgay and A. Possenti for useful discussions about the relation between dispersion measure and equivalent hydrogen column of the interstellar medium. This work was partially supported by the Italian Space Agency (ASI) and the Ministero della Istruzione, della Universitá e della Ricerca (MIUR). 


\section{References}

Augello, G., Iaria, R., Robba, N. R., et al. 2003, ApJ, 596, L63 Boella, G., Butler, R. C., Perola, G. C., et al. 1997, A\&AS, 122, 299 Bradt, H., Naranan, S., Rappaport, S., \& Spada, G. 1968, ApJ, 152, 1005

Frontera, F., Costa, E., dal Fiume, D., et al. 1997, A\&AS, 122, 357

Gursky, H., Gorenstein, P., \& Giacconi, R. 1967, ApJ, 150, 75

Hasinger, G., \& van der Klis, M. 1989, A\&A, 225, 79

Iaria, R., Di Salvo, T., Robba, N. R., et al. 2004, ApJ, 600, 358

Iaria, R., Spanó, M., Di Salvo, T., et al. 2005, ApJ, 619, 503

in 't Zand, J. J. M., Verbunt, F., Strohmayer, T. E., et al. 1999, A\&A, 345,100

Krolik, J. H., McKee, C. F., \& Tarter, C. B. 1981, ApJ, 249, 422

Langmeier, A., Sztajno, M., Truemper, J., \& Hasinger, G. 1985, SSRv, 40,367

Manzo, G., Giarrusso, S., Santangelo, A., et al. 1997, A\&AS, 122, 341
Parmar, A. N., Martin, D. D. E., Bavdaz, M., et al. 1997, A\&AS, 122, 309

Predehl, P., \& Schmitt, J. H. M. M. 1995, A\&A, 293, 889

Reynolds, C. S., \& Fabian, A. C. 1995, MNRAS, 273, 1167

Singh, K. P., \& Apparao, K. M. V. 1994, ApJ, 431, 826

Schulz, N. S., Hasinger, G., \& Truemper, J. 1989, A\&A, 225, 48

Taylor, J. H., \& Cordes, J. M. 1993, ApJ, 411, 674

Titarchuk, L. 1994, ApJ, 434, 570

Titarchuk, L., \& Lyubarskij, Y. 1995, ApJ, 450, 876

van der Klis, M. 2000, ARA\&A, 38, 717

Verner, D. A., \& Ferland, G. J. 1996, ApJS, 103, 467

Verner, D. A., Ferland, G. J., Korista, K. T., \& Yakovlev, D. G. 1996, ApJ, 465, 487

White, N. E., Stella, L., \& Parmar, A. N. 1988, ApJ, 324, 363

Wijnands, R., van der Klis, M., \& van Paradijs, J. 1998, Proc. IAU Symp., ed. Katsuji Koyama, Shunji Kitamoto, \& Masayuki Itoh (Dordrecht: Kluwer Academic), 188, 370 